\title{
POLY(ETHYLENE-CO-METHYL ACRYLATE)/POLY(CAPROLACTONE) TRIOL BLENDS FOR DRUG DELIVERY SYSTEMS: CHARACTERIZATION AND DRUG RELEASE
}

\author{
Luiz A. Kanis* \\ Universidade do Sul de Santa Catarina, Av. José Acácio Moreira, 787, 88704-900 Tubarão - SC, Brasil \\ Valdir Soldi \\ Departamento de Química, Universidade Federal de Santa Catarina, CP 476, 88040-900 Florianópolis - SC, Brasil
}

Recebido em 16/2/11; aceito em 5/7/11; publicado na web em 19/8/11

\begin{abstract}
Poly(ethylene-co-methyl acrylate) (EMA) and poly (caprolactone) triol (PCL-T) blends, a biodegradable aliphatic polyester with low molecular weight and moderate water solubility containing diltiazem hydrochloride (DZ) were studied in terms of the thermal and morphological properties, and drug release mechanism. An increase in the PCL-T content in the EMA/PCL-T/DZ films decreased the degree of DZ crystallinity. Drug release from these films is temperature-dependent, and it is possible to modify the drug release rate by adjusting the EMA/PCL-T composition of the blends. The mechanism of drug release is governed by PCL-T melting and PCL-T leaching from EMA matrix.
\end{abstract}

Keywords: blends; poly(caprolactone) triol; drug release.

\section{INTRODUCTION}

The development of polymeric blends is an alternative means to obtain new materials for pharmaceutical application. Polymeric properties such as elasticity, softness, tensile strength, biodegradability and drug diffusivity can be modified by blending. ${ }^{1-5}$ The properties of a broad range of polymers used in the pharmaceutical industry were modified by blending processes, such as cellulose derivatives, polysaccharides, poly(ethylene oxide), poly acrylic acids, poly(ethylene-co-vinyl acetate) (EVA), acrylates, silicones, poly(caprolactones) (PCL)..$^{6-14}$

Interest in polymer blending for the biomedical area, mainly to control drug release, has increased considerably due to the small range of polymers available for in vivo use. The properties of biodegradable polymers, such as PCL, and non-biodegradable but biocompatible polymers, such as EVA and acrylates, are modified for use in drug delivery systems. ${ }^{15-17}$ Water permeability and hydration ability can be modified by increasing the hydrophilic polymer content in a hydrophilic-hydrophobic polymeric blend, thus modifying the drug release profile and film erosion. However, in many cases, this is also associated with the disadvantage of a reduction in the mechanical strength of the polymeric film as a result of a decrease in the polymer crystallinity degree. ${ }^{5,18}$ Another alternative is the addition of a polymer fusible at body temperature to a biodegradable polymeric blend, which can increase matrix porosity through polymer leaching, thereby increasing drug release and degradation rates. ${ }^{19}$

Poly(caprolactone) blends have been extensively studied, but there are only a small number of studies specifically on poly(caprolactone triol) (PCL-T) blends. PCL-T is a biodegradable aliphatic polyester with low molecular weight, moderate water solubility and melting temperature close $33{ }^{\circ} \mathrm{C} .{ }^{19}$ There are three hydroxyl groups to which the ability to plasticize some natural polymers, such as soy protein and cellulose acetate, is attributed. ${ }^{20,21}$ Meier et al. ${ }^{7}$ showed that the addition of liquid PCL-T (Mw $300 \mathrm{~g} / \mathrm{mol}$ ) to cellulose acetate films modulates drug permeability through a decrease in the polymer-polymer interac-

*e-mail: luiz.kanis@unisul.br tions (plasticizing effect) and the formation of small pores and channels through PCL-T dissolution.

Blends such as poly(ethylene-co-methyl acrylate (EMA) and semi-solid PCL-T (600 g/mol) have also been studied. ${ }^{21}$ EMA is an elastomeric copolymer composed of ethylene and methyl acrylate units, which are crystalline and amorphous, respectively. ${ }^{22}$ EMA, just like EVA, has good mechanical properties for the development of controlled-drug release systems. ${ }^{23}$ Kanis et al. ${ }^{21}$ showed that poly(caprolactone triol) added to the EMA matrix produces immiscible films without significantly changing the elasticity modulus; moreover, the PCL-T melting point in the blend remained close to $30^{\circ} \mathrm{C}$.

The main objective of this study was to investigate the effect of PCL-T content in the EMA matrix on drug release of hydrophilic drug. PCL-T may be used to control the drug release at body temperature $\left(37^{\circ} \mathrm{C}\right)$ from EMA films by leaching process without causing toxicity due to its biodegradability. Diltiazem hydrochloride was used as model drug. The matrix was studied in terms of drug crystallinity degree; film morphology; diltiazem hydrochloride release profiles; and temperature effect on drug release.

\section{EXPERIMENTAL}

\section{Materials}

Poly(ethylene-co-methyl acrylate) (EMA) copolymer with $29.0 \%$ methyl acrylate content and poly(caprolactone triol), $M_{n}=600 \mathrm{~g}$ $\mathrm{mol}^{-1}$ were obtained from Sigma-Aldrich Chem. Co. (St. Louis, USA). Chloroform was purchased from Vetec S.A. (Rio de Janeiro, Brazil). Both the polymers and chloroform were used without further purification. Pharmaceutical grade diltiazem hydrochloride (DZ) was obtained from Sigma-Aldrich Chemie Co (Steinhein, Germany) and was also used without further purification.

\section{Preparation of EMA/PCL-T films}

Films were prepared by solvent casting method. Different amounts of EMA, PCL-T and DZ (80/0/20; 70/10/20; 60/20/20; $50 / 30 / 20 \%, w / w / w)$ were dissolved in $10 \mathrm{~mL}$ of chloroform and 
maintained in sealed vials under agitation at $40{ }^{\circ} \mathrm{C}$ for $2 \mathrm{~h}$.

The polymer/drug solutions were poured onto a Teflon-coated plate (petri dish) for the solvent evaporation at room temperature. The membrane was removed from the plate, dried for $24 \mathrm{~h}$ at room temperature, and stored in a vacuum desiccator until analysis. The average thickness of the membranes was determined after measurements at different points in the film using a micrometer (Mytotoyo, Japan).

\section{Differential scanning calorimetry (DSC)}

DSC curves of the EMA/PCL-T blends containing DZ were obtained on a differential scanning calorimeter (DSC 50, Shimadzu) by heating the samples from -25 to $200{ }^{\circ} \mathrm{C}$, at a heating rate of $10{ }^{\circ} \mathrm{C}$ $\mathrm{min}^{-1}$. The average sample size was $8 \mathrm{mg}$ and the nitrogen flow-rate was $50 \mathrm{~cm}^{3} \mathrm{~min}^{-1}$. Indium $\left(156.6^{\circ} \mathrm{C}\right)$ and zinc $\left(419.5^{\circ} \mathrm{C}\right)$ standards were used for the calibration. The relative crystallinity degree $\left(\mathrm{X}_{c}\right)$ of diltiazem hydrochloride in the EMA/PCL-T films was calculated by the Equation 1:

$$
\mathrm{X}_{\mathrm{c}}(\%)=\Delta \mathrm{H} / \Delta \mathrm{H}_{\mathrm{m}} \cdot 100
$$

where $X_{c}(\%)$ is the relative percent degree of crystallinity, $\Delta H$ is the melting enthalpy of DZ in the blend, $\Delta \mathrm{H}_{\mathrm{m}}$ is the melting enthalpy of pure DZ.

\section{Morphological analysis}

A scanning electron microscope (Philips XL 30) was used to observe the morphology of the surface and cross-section of the EMA/ PCL-T/DZ membranes coated with a thin layer of gold. To observe the cross-section, the samples were fractured under liquid nitrogen.

\section{Drug release}

The drug release experiments were performed using an Ética 310 dissolution test system (Nova Ética S.A., Brazil). Drug release from the films was determined by using the paddle over disk assembly, adapted from the United States Pharmacopeia.

The dry films with $18 \mathrm{mg}$ of drug content and known thickness were cut into circular-shaped samples $\left(7.07 \mathrm{~cm}^{2}\right)$ and fixed to an acrylic plate. The plate was immersed in $800 \mathrm{~mL}$ of phosphate buffer solution ( $\mathrm{pH}=7.4$ ) (to maintain sink conditions). All experiments were maintained at a temperature of $37.0 \pm 0.5^{\circ} \mathrm{C}$ or $25 \pm 0.5^{\circ} \mathrm{C}$. The paddle was positioned at a distance of $2.5 \mathrm{~cm}$ from the surface of the acrylic plate and set to rotate at a speed of $60 \mathrm{rpm}$. Samples were taken periodically at predetermined time intervals and after suitable dilution with the buffer solution, the total drug released was obtained against a predetermined calibration curve ( 5 dilutions between $0.0020-0.0230 \mathrm{mg} / \mathrm{mL}$ ) using a UV-vis spectrophotometry at $\lambda_{\max }$ of $239 \mathrm{~nm}$, on a Hitachi 2010 double-beam UV-visible spectrophotometer. The spectrophotometric method was linear $(\mathrm{r}=$ $0,99994)$, precise $(\mathrm{RSD}=1,9 \%)$, exact $(98,1 \pm 2,4 \%)$ and specific to assay DZ in buffer solution in the PCL-T and EMA presence. The quantification and detection limit were 0,0009 and $0,0003 \mathrm{mg} /$ $\mathrm{mL}$, respectively. After each sample was taken, an equal volume of solution was added to the dissolution medium to maintain a constant volume.

\section{Drug release mechanism}

The mechanisms of transport through polymeric systems were described by the following equation:

$$
M / M_{\infty}=b t^{n}
$$

where $M_{t}$ is the amount of the drug released at time $t, M_{\infty}$ is the overall amount of the drug, $b$ is a constant incorporating the structural and geometric characteristics of the controlled release device, and $n$ is the release exponent indicative of the drug release mechanism. For a system of known geometry, $n=0.5$ means Fickian diffusion, $0.5<>1.0$ non-Fickian diffusion, and $n=1.0$ Case II diffusion. ${ }^{24,25}$ The diffusion exponent (n) was calculated from the fitted linear regression lines of double logarithmic plots of fractional drug released over time. The slope was taken from the linear portion of the graph (a maximum of $60 \%$ of the drug was released).

\section{Polymer weight loss $(\%)$}

After drug release studies, the films were dried under low pressure at $50{ }^{\circ} \mathrm{C}$ temperature and the polymer weight loss after drug release test was determined by the Equation 3:

$$
\text { Polymer weight loss (\%): (FW - FWA).100/FW }
$$

where: $\mathrm{Fw}=$ initial film weight - total drug content; $\mathrm{FWA}=$ film weight after drug released at $24 \mathrm{~h}$ - initial drug content - total drug released at $24 \mathrm{~h})$.

\section{RESULTS AND DISCUSSION}

\section{Thermal characterization of EMA/PCL-T/DZ films}

The melting point peak and crystallinity degree obtained from DSC curve (Figure 1S, supplementary material) of the DZ as well as the EMA/PCL-T/DZ films are shown in Table 1.

Table 1. The melting point peak (MP), melting enthalpies $\left(\Delta \mathrm{H}_{\mathrm{m}}\right)$ and relative crystallinity degree $\left(X_{c}\right)$ of diltiazem hydrochloride in the EMA/PCL-T films

\begin{tabular}{lccc}
\hline \multirow{2}{*}{ EMA/PCL-T/Drug $(\%)$} & \multicolumn{3}{c}{ Diltiazem hydrochloride } \\
& $\Delta \mathrm{H}_{\mathrm{m}}(\mathrm{J} / \mathrm{g})$ & $\mathrm{MP}\left({ }^{\circ} \mathrm{C}\right)$ & $\mathrm{X}_{\mathrm{c}}(\%)$ \\
\hline $0 / 0 / 100$ & -67.33 & 216.1 & 100 \\
$80 / 0 / 20$ & -23.65 & 212.9 & 35.1 \\
$70 / 10 / 20$ & -15.46 & 203.9 & 23.0 \\
$60 / 20 / 20$ & $\mathrm{ndt}$ & 184.7 & $\mathrm{ndt}$ \\
$50 / 30 / 20$ & $\mathrm{ndt}$ & 171.8 & $\mathrm{ndt}$ \\
\hline
\end{tabular}

Ndt: not determined

The DSC plots showed a shift in the thermal transitions of DZ in the films. A gradual reduction in the $\Delta \mathrm{H}_{\mathrm{m}}$ and melting point of DZ was observed with an increase in the PCL-T content in the blend. The relative degree of DZ crystallinity was not determined for the blends with 30 and $40 \%$ of PCL-T; however, it is expected to be close to $0 \%$ given the strong effect of PCL-T on the DZ crystallization. The crystallinity of DZ, a hydrophilic drug, was affected by the EMA polymer and an additional effect of the PCL-T was observed. These results show that drug characteristics and EMA/PCL-T ratio are factors which can be used to control drug crystallinity in the polymeric matrix and consequently the drug diffusion properties. ${ }^{16,26,27}$

\section{Morphological analysis of EMA/PCL-T/DZ}

SEM micrographs of the surfaces of EMA/PCL-T/DZ films are shown in Figure 1. 


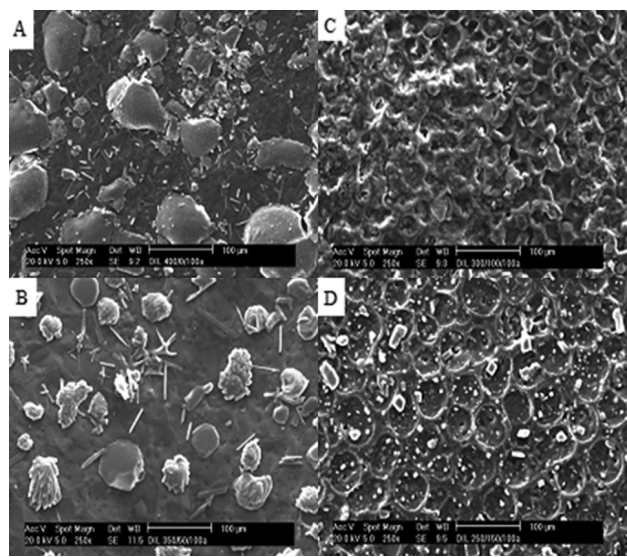

Figure 1. SEM micrographs of EMA/PCL-T/DZ film surfaces - 80/0/20 (A), 70/10/20 (B), 60/20/20 (C) and 50/30/20 (D)

Kanis et al. ${ }^{21}$ reported that EMA films have a homogeneous surface and no apparent porosity in the cross-sections. The EMA/PCL-T/DZ (80/0/20) micrograph (Figure 1A) shows DZ crystals spread across the surface and shows porosity in the cross-section related to the presence of DZ in the film (Figure $2 \mathrm{~S} \mathrm{E}$, supplementary material). The quantity of crystal on the surface may be associated with the degree of DZ crystallinity observed in the DSC results. The EMA/PCL-T/DZ 80/0/20 and 70/10/20 films (Figures 1A and 1B) showed a great number of DZ crystals on the surface and the DSC analysis showed crystallinity degrees of 35.1 and $23.0 \%$, respectively. However, the $60 / 20 / 20$ and $50 / 30 / 20$ films showed a degree of DZ crystallinity close to $0 \%$ as a result of the decrease in the amount of crystals on the film surface and an increase in the drug solubility in the polymeric matrix due to the presence of the PCL-T (Figures 1C and 1D). In addition, phase separation was observed on the surface of the 60/20/20 and 50/30/20 films (Figures 1C, 1D), showing domains rich in PCL-T. The same behavior is observed in the cross-section of the blends however from the blend 70/10/20 (Figures 2SF, 2SG, 2SH, supplementary material).

\section{Drug release from EMA/PCL-T films}

Figure 2 showed the DZ release profiles for the EMA/PCL-T/ DZ films at $37^{\circ} \mathrm{C}$.

The profiles showed a fast and low DZ content release from EMA/ PCL-T/DZ 80/0/20 and 70/10/20 films in the first few hours. In the next $24 \mathrm{~h}$, no significant differences were observed in the amount of DZ released. The EMA copolymer is hydrophobic and does not swell in water, ${ }^{23}$ a characteristic which restricts the drug partition from the film to the phosphate buffer bulk. Thus, the amount of drug released from the film in the first few hours can be attributed to the drug present

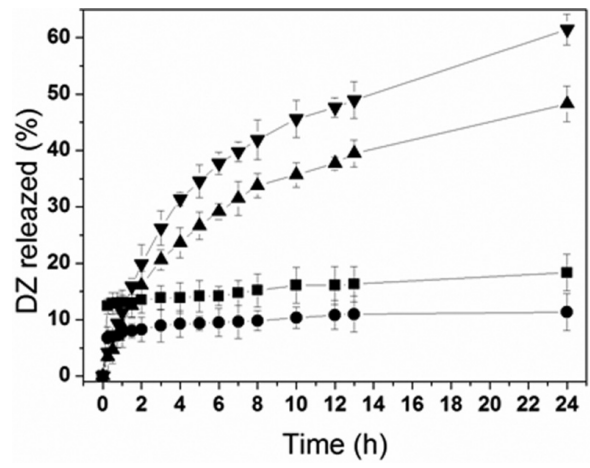

Figure 2. DZ release profiles for EMA/PCL-T/DZ films: (匹) 80/0/20;

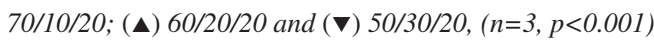

at the film surface, as observed in the SEM micrographs (Figure 1). An increase in the PCL-T content to $10 \%$ does not promote significant changes in the drug release profile $(\mathrm{p}<0.001)$.

The profiles for the DZ release from the EMA/PCL-T/DZ60/20/20 and 50/30/20 films showed that an increase in PCL-T content in the film leads to a lower drug release rate during the first hour, associated with a lower amount of drug on the film surface, as observed in the SEM micrographs, although there is an increase in the total amount of drug released. In the case of these films, this increase could be associated with the PCL-T melting at the experimental temperature $\left(37^{\circ} \mathrm{C}\right)$, which could lead to an increase in the drug diffusion from the EMA films. In order to better analyze the temperature effect on the drug release from the films with PCL-T, drug release profiles at 25 and $37{ }^{\circ} \mathrm{C}$ were obtained from EMA/PCL-T/DZ 80/0/20 (no PCL-T) and 60/20/20 and the results are shown in Figures 3 and 4.

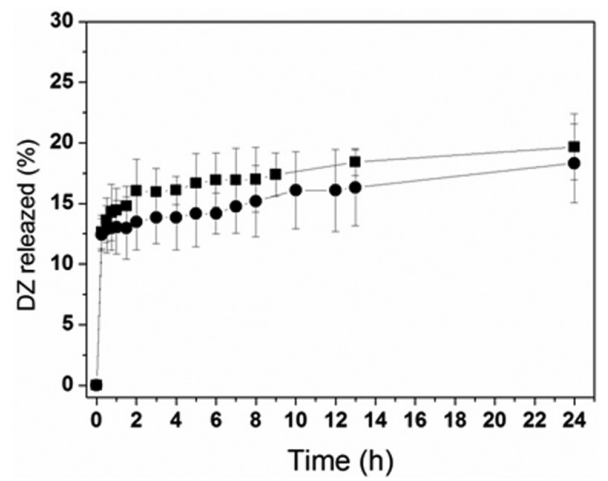

Figure 3. DZ release from EMA/PCL-T/DZ 80/0/20 film at $25^{\circ} \mathrm{C}(\bullet)$ and $37^{\circ} \mathrm{C}(\mathbf{\square}),(n=3, p<0.001)$

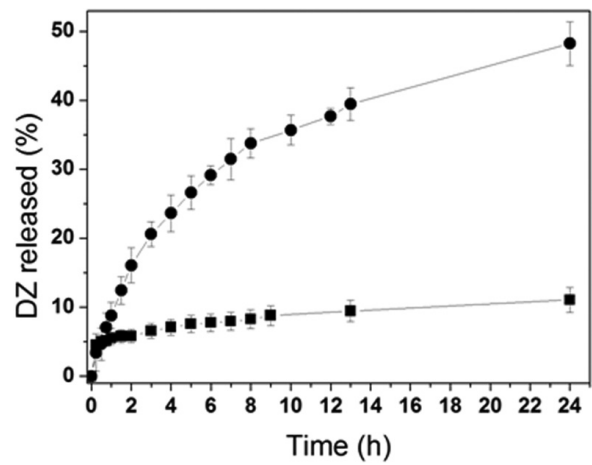

Figure 4. DZ release from EMA/PCL-T/DZ 60/20/20 film at $25^{\circ} \mathrm{C}$ (匹) and $37^{\circ} \mathrm{C}(\bullet),(n=3, p<0.001)$

The profiles of the DZ release from the EMA/PCL-T/DZ 80/0/20 (no PCL-T content) films at 25 and $37{ }^{\circ} \mathrm{C}$ (Figure 3) showed a low amount of DZ released during the experimental time and an increase in temperature did not lead to a significant change in this behavior. These results reinforced what was explained, that the amount of drug released from the films without PCL-T is mainly that on the surface of the film.

Figure 4 shows the profiles of DZ release from EMA/PCL-T/DZ $60 / 20 / 20$ (20\% of PCL-T content) at 25 and $37^{\circ} \mathrm{C}$. The total DZ released at $24 \mathrm{~h}$ from the EMA/PCL-T/DZ 60/20/20 film at $25^{\circ} \mathrm{C}$ was lower than that observed for the $80 / 0 / 20$ film attributed to reduced quantity of drug crystal spread on the film surface and increase in drug solubility on the matrix. When the temperature was increased to $37{ }^{\circ} \mathrm{C}$, an increase in the drug delivery from the film was observed. There was a significant influence of temperature and PCL-T content on the drug release, which could be explained not by plasticizer effect of PLC-T, as Meier et al. ${ }^{21}$ 
observed for cellulose acetate membranes, but by PCL-T melting. The increase in drug released from films containing PCL-T at $37^{\circ} \mathrm{C}$ can be attributed to solid-liquid transitions of PCL-T, which promotes drug release from the film by increasing the drug diffusion across the film and by PCL-T leaching. The film without PCL-T exhibited no polymer weight loss at 25 and $37^{\circ} \mathrm{C}$ (Table 2) because EMA polymer is neither biodegradable nor water soluble. The increase in PCL-T content into the film promotes a small increase in polymer weight loss at $25{ }^{\circ} \mathrm{C}$, although a significant weight loss was observed at $37^{\circ} \mathrm{C}$, which explains the great drug release from these films at this temperature.

Table 2. Diffusion mechanism obtained applying the Power Law, total DZ released and total loss weight at $24 \mathrm{~h}$ from EMA/PCL-T/DZ films at $37{ }^{\circ} \mathrm{C}$

\begin{tabular}{lccccc}
\hline EMA/ & \multirow{2}{*}{$\mathrm{DZ}_{24 \mathrm{~h}}(\%)$} & \multicolumn{2}{c}{ Mechanism } & \multicolumn{2}{c}{ Polymer loss weight $(\%)$} \\
PCL-T/DZ & & $n$ & $\mathrm{r}$ & $25^{\circ} \mathrm{C}$ & $37^{\circ} \mathrm{C}$ \\
\hline $80 / 0 / 20$ & $20.4 \pm 3.2$ & $\mathrm{ndt}$ & $\mathrm{ndt}$ & $0.04 \pm 0.03$ & $0.03 \pm 0.02$ \\
$70 / 10 / 20$ & $12.8 \pm 3.7$ & $\mathrm{ndt}$ & $\mathrm{ndt}$ & $0.31 \pm 0.13$ & $0.42 \pm 0.14$ \\
$60 / 20 / 20$ & $48.22 \pm 3.6$ & 0.71 & 0.9952 & $0.62 \pm 0.21$ & $3.83 \pm 1.10$ \\
$50 / 30 / 20$ & $61.40 \pm 2.76$ & 0.70 & 0.9982 & $0.82 \pm 0.31$ & $5.67 \pm 1.42$ \\
\hline
\end{tabular}

$\mathrm{n}$ : exponential value applying Power Law; r: correlation coefficient; ndt: not determined

Table 2 shows the diffusion mechanism obtained by applying the Power Law, total Dz released and total weight loss at $24 \mathrm{~h}$ from EMA/PCL-T/DZ films at $37^{\circ} \mathrm{C}$.

It was not possible to determine the mechanism of drug release from the EMA/PCL-T/DZ 80/0/20 and 70/10/20 films due to the small quantity of drug released in the first hour. In general, for hydrophobic polymers such as EMA, the drug diffusion is affected mainly by the diffusion coefficient across the polymer, water uptake kinetics and the presence of filler and additives. ${ }^{17,28-30}$ In these cases, the drug released is governed only by the diffusion coefficient because EMA is a nonwater-swellable polymer and the low DZ solubility in the polymeric matrix may explain the low content of $\mathrm{DZ}$ released. The $n$ values of EMA/PCL-T/DZ 60/20/20 and 50/30/20 (Table 2) were obtained by applying the Power Law 0,7 . These results indicate that DZ release from these films is governed by an anomalous transport where a superposition of diffusional and relaxational release occurs. The presence of PCL-T in the EMA film leads to an increase in the diffusion of the DZ out of the matrix, due to the improved polymeric chain relaxation caused by the PCL-T melting followed by PCL-T leaching at $37^{\circ} \mathrm{C}$. Thus, the incorporation of biodegradable PCL-T to biocompatible copolymer EMA becomes an alternative to modify drug release and can be used to obtain human body implantable devices.

\section{CONCLUSION}

The results of this study show that the increase in PCL-T into the EMA/DZ matrix decreases the crystallinity degree of DZ. Thus, the EMA/PCL-T ratio is a factor that can be used to control drug crystallinity in the polymeric matrix and, consequently, the drug release properties.

The drug release from these films is dependent on PCL-T melting at $37{ }^{\circ} \mathrm{C}$, followed by leaching. So, it is possible to modify the drug release rate by adjusting the composition of EMA/PCL-T in the blends. The results indicate that the EMA/PCL-T blends are good candidates for the development of implantable drug devices.

\section{SUPPLEMENTARY MATERIAL}

The DSC curves (Figure 1S) and MEV from cross section of the EMA/PCL-T/DZ films (Figure 2S) are available at supplementary material in http://quimicanova.sbq.org.br, with free access.

\section{ACKNOWLEDGEMENTS}

The authors acknowledge CAPES (Coordenação de Aperfeiçoamento de Pessoal de Nível Superior), CNPq (Conselho Nacional de Desenvolvimento Científico e Tecnológico), UFSC (Universidade Federal de Santa Catarina) and UNISUL (Universidade do Sul de Santa Catarina) for financial support.

\section{REFEREN CES}

1. Nyamweya, N.; Hoag, S. W.; Pharm. Res. 2000, 17, 625.

2. Lao, L. L.; Venkatraman, S. S.; Peppas, N. A.; Eur. J. Pharm. Biopharm. 2008, 70, 796

3. Bajpai , A. K.; Shukla, S. K.; Bhanu, S.; Kankane, S.; Prog. Polym. Sci. 2008, 33, 1088.

4. Khan, I. A.; Patravale, V. B.; Drug Dev. Ind. Pharm. 2005, 31, 59.

5. Lyu, S. P.; Sparer, R.; Hobot, C.; Dank, K.; J. Controlled Release 2005, 102, 679.

6. Shi, R.; Zhu, A.; Chen, D.; Jiang, X.; Xu, X.; Zhang, L.; Tian, W.; J. Appl. Polym. Sci. 2009, 115, 346.

7. Meier, M. A.; Kanis, L. A; Soldi, V.; Int. J. Pharm. 2004, 278, 99.

8. MacDonald, P. F.; Lyons, J. G.; Geever, L. M.; J. Mater. Sci. 2010, 45, 1284.

9. Kanis, L. A.; Viel, F. C.; Crespo, J. S.; Bertolino, J. R.; Pires, A. T. N.; Soldi, V.; Polymer 2000, 41, 3303.

10. Mariani, P. D. S.; Allganer, K.; Oliveira, F. B.; Cardoso, E. J. B. N.; Mei, L. H. I.; Polym. Test. 2009, 28, 824.

11. Li, J.; Suo, J.; Deng, R.; J. Reinf. Plast. Comp. 2010, 29, 618.

12. Natu, M. V.; Gil, M. H.; Souza, H. C.; J. Supercrit. Fluids 2008, 47, 93.

13. Moly, K.A.; Bhagawan, S. S.; George, S. C.; Thomas, S.; J. Mater. Sci. 2007, 42, 4552.

14. Li, J.; Barrow, D.; Howell, H.; Kalachandra, S.; J. Mater. Sci. 2009, 21,583.

15. Lyons, J. G.; Blackie, P.; Higginbotham, C. L.; Int. J. Pharm. 2008, 351, 201.

16. Liao, H. T.; Wu, C. S.; Mater. Sci. Eng., A 2009, 515, 207.

17. Ha, C. S.; Cho, W. J.; Prog. Polym. Sci. 2002, 27, 759.

18. Cheng, L.; Lei, L.; Shengrong, G.; Int. J. Pharm. 2010, 387, 129.

19. Kanis, L. A.; Generoso, M.; Soldi, V.; Lat. Am. J. Pharm . 2007, 26, 700.

20. Schmidt, V.; Giacomelli, C.; Soldi, M. S.; Soldi, V.; Macromol .Symp. 2005, 229, 127.

21. Meier, M. M.; Kanis, L. A.; Lima, J. C.; Pires, A. T. N.; Soldi, V.; Polym. Adv. Technol. 2004, 15, 593.

22. Kundu , P. P.; Polymer 1996, 37, 2423.

23. Kanis, L. A., Generoso, M.; , Méier, M. M.; Pires, A. T. N.; Soldi, V.; Eur. J. Pharm. Biopharm. 2005, 60, 383.

24. Peppas, N. A.; Pharm. Acta Helvetica 1985, 60, 110.

25. Higuchi, T.; J. Pharm. Sci. 1961, 50, 874.

26. Florence, A. T.; Attwood, D.; Physicochemical Principles of Pharmacy, $3^{\text {rd }}$ ed., Creative Print \& Design: London, 2003.

27. Lipp, R.; J. Pharm. Pharmacol. 1998, 50, 1343.

28. Kandavilli, S.; Nair, V.; Panchagnula, R.; Pharmaceutical Technology 2002, $26,62$.

29. Pankova, Y. N.; Shchegolikhin, A. N.; Iordanskii, A. L.; Zhulkina, A. L.; Ol'khov, A. A.; Zaikov, G.E.; J. Mol. Liq. 2010, 156, 65.

30. Cheng, L.; Guo, S.; Wu, W.; Int. J. Pharm. 2009, 377, 112. 